\title{
Defusing the new drug-resistant TB time bomb
}

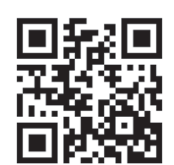

A nationally co-ordinated strategy is urgently needed to avoid the discharge of highly contagious 'therapeutically destitute' tuberculosis (TB) patients back into a careless void, with uniquely tailored solutions including home-based or community care, plus multidisciplinary teams in modernday sanatoria.

This appeal was made at the annual National TB Conference in Durban in midJune by Prof. Keertan Dheda, arguably the country's leading expert on drug-resistant (DR) TB. He warned that fast-spreading, highly DR TB will further discourage entry to the healthcare professions, aggravating the existing staff shortage crisis and crippling South Africa (SA)'s ability to reduce its quadruple burden of disease.

Dheda, Professor of Medicine and Head of Pulmonology at the University of Cape Town (and also one of the most published and cited TB academics in the country), estimates that several thousand healthcare workers (HCWs) are currently TB-infected (an annual infection rate of 2 - 3\%). Acknowledging that successfully combating DR TB will be a 'massive undertaking', he appealed for greater emphasis on safety by the national health department via the Occupational Health and Safety Act and creation of user-friendly and closely supervised TB patient-triaging policies - in tandem with better environmental and personal control measures.

\section{When a patient with infectious \\ DR TB is admitted to such a} setting, up to $50 \%$ of the patients exposed on that ward can become infected within 24 hours.

\section{Hospitals DR TB \\ 'infection palaces'}

Across SA (as in many other parts of the world), patients are admitted to large hospital wards or spend time in crowded outpatient waiting areas - with little or no ventilation. Modelling studies have shown that when a patient with infectious DR TB is admitted to such a setting, up to $50 \%$ of the patients exposed on that ward can

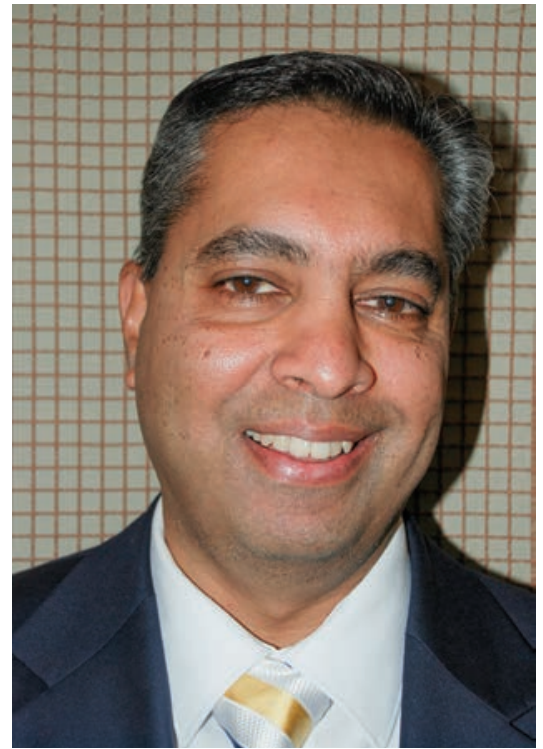

Prof. Keertan Dheda, top expert on drug-resistant TB. Picture: Chris Bateman.

become infected - within 24 hours. Dheda's call for a more effective overall strategy underscores repeated ongoing appeals by the Treatment Action Campaign and Section 27 to declare DR TB a national emergency and for an aggressive push by national health chiefs to beef up infection control policies in hospitals, especially through monitoring and support of the more laggardly provincial health departments. Dheda said that with the country's heavy patient load and resource constraints, home-based or communitybased care - with suitable logistical support for a specific category of patients, including ventilation grants - would be a pragmatic way forward. 'They have families, they have to work, some are even driving taxis, he emphasised. However, there was also a category of DR TB patients who were unable to work owing to their advanced illness or family circumstances.

\section{Multidisciplinary sanatoria and safe palliation needed}

Easily accessible long-term inpatient facilities with multidisciplinary teams - the equivalent of modern-day sanatoria - were urgently needed to help these people. 'We need them [such sanatoria] and we need them right now' he emphasised. Also, specially tailored palliative care facilities were required to deal with the alarming number of therapeutically destitute TB patients discharged from hospital. 'They do not die immediately (the median survival rate is two years as we recently showed in a paper in the Lancet) and often go back to a district hospital where there is more disease transmission. They also need to be able to die with dignity - you cannot put them in a standardised hospice setting because of the extraordinary infection control requirements!' Creative discussions with the Hospice and Palliative Care Association of Southern Africa could prove fruitful, Dheda suggested. Among other things, specially trained nurses with highly effective protection measures were required. Dheda said he was aware of some rare exceptions to the national paucity of specially tailored palliative care facilities: a two-bed facility run by Médecins Sans Frontières in Khayelitsha and another larger one at Nelspoort, also in the Western Cape. While valuable as models, they were mere drops in the required caregiving ocean, with the Nelspoort facility 'not working very well because it's too far away from anything' Dheda was among a handful of local scientists whose research has helped define TB treatment failure (failing to respond to treatment or culture-converting within 12 months).

The WHO warned that any government doubt and inaction could spawn a full-blown XDR epidemic.

\section{HCW infections - alarming findings}

Seminal work on local HCW TB infection (published in 2010 and in collaboration with researchers at the University of KwaZulu-Natal) involved a 6-year study at King George V Hospital in Durban, where Dheda and his fellow researchers uncovered 250 HCWs with extensively drug-resistant (XDR) and multidrug-resistant (MDR) TB, who had an infection rate six times higher than the general population (Table 1). In another study recently published in the Lancet, there was an $11 \%$ rate of favourable 5 -year outcomes (cured or completing treatment) among patients with XDR TB, with mortality at $75 \%$. Notably, half of 
Table 1. MDR- and XDR-infected HCWs, KZN Province

\begin{tabular}{llll}
\hline & HCWs & General population & $\begin{array}{l}\text { Incidence rate ratio } \\
(\mathbf{9 5 \%} \text { CI) }\end{array}$ \\
\hline $\begin{array}{l}\text { Annual MDR or XDR TB } \\
\text { incidence } \\
(/ 100 \text { 000) }\end{array}$ & 66.8 & 11.7 & $\begin{array}{l}5.71 \\
(4.96-6.69)\end{array}$ \\
$\begin{array}{l}\text { Annual MDR TB } \\
\text { incidence } \\
(/ 100 \text { 000) }\end{array}$ & 62.3 & 10.7 & 5.82 \\
$\begin{array}{l}\text { Annual XDR TB incidence } \\
(/ 100 \text { 000) }\end{array}$ & 4.5 & 1.04 & $(5.03-6.87)$ \\
$\begin{array}{l}\text { MDR = multidrug-resistant; XDR = extensively drug-resistant; HCWs = healthcare workers; KZN = KwaZulu-Natal; } \\
\text { CI = confidence interval; TB = tuberculosis. }\end{array}$ & & 4.33 \\
\end{tabular}

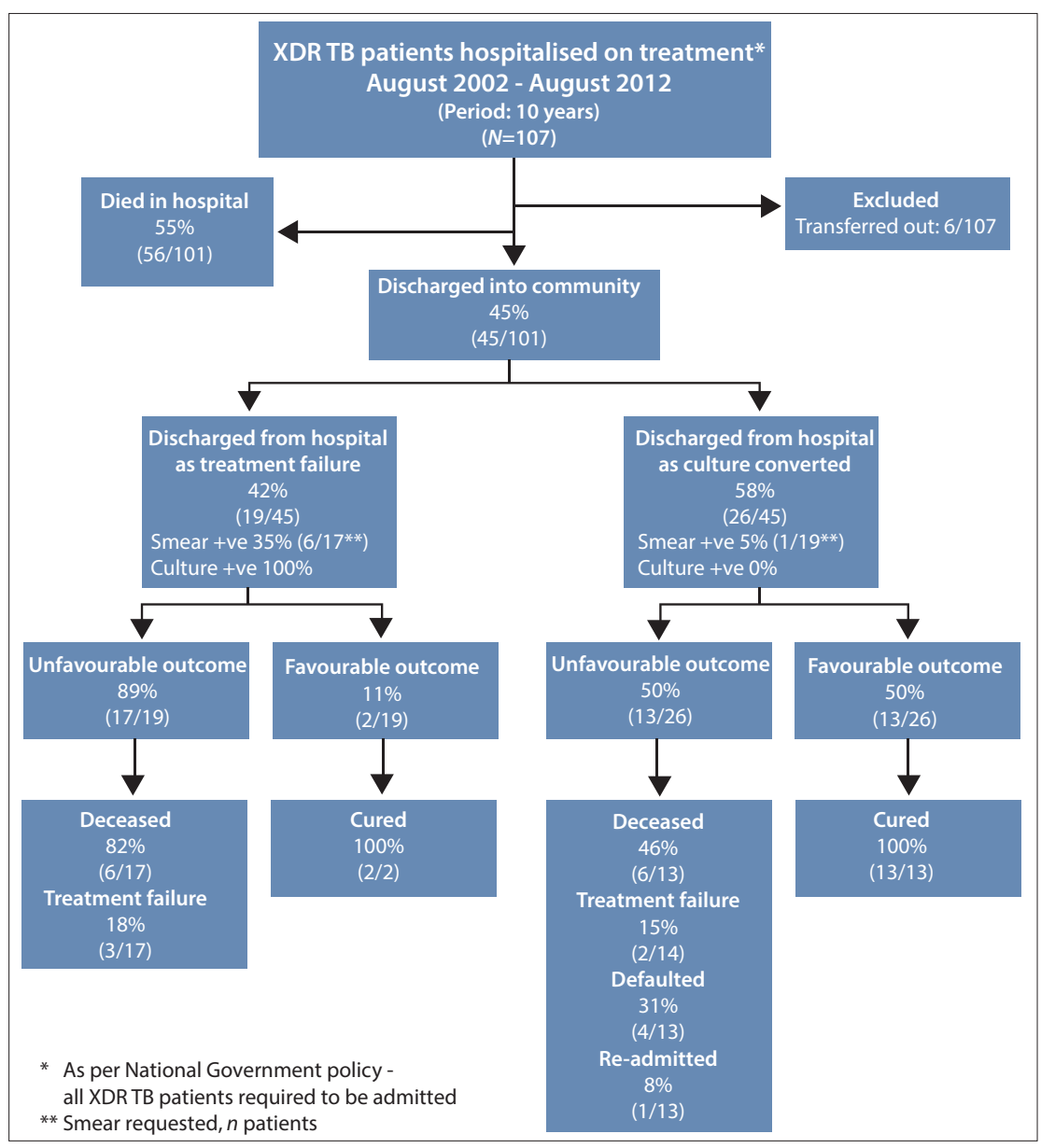

Fig. 1. Mortality and outcome after hospital discharge for XDR TB patients, August 2002 - August 2012. $(X D R=$ extensively drug-resistant; $T B=$ tuberculosis. $)$

them were HIV-negative. It was also here that his team discovered just how many incurable patients were being discharged into the community - of $107 \mathrm{XDR}$ patients tracked, half made it to discharge, and only half of this (discharged) cohort were 'culture converters' (treatment responders) (Fig. 1). 'So the hopeless cases leave with no palliative care or long-stay community facilities available. They just go home, he similar findings, suggesting that thi happening on a wide scale: 'it's not just sporadic', Dheda emphasised.

Nationally, an estimated 600-1000 known XDR cases annually are being added to the current known tally of 8000 cases of DR TB, with the World Health Organization (WHO) warning that any government doubt and inaction could spawn a full-blown XDR epidemic. By way of comparison (albeit with a First-World country), the USA treated approximately 100 cases of MDR TB last year.

Dheda said that major improvements in laboratory capacity, infection control, the performance of the TB control programme, and treatment regimens for both drugsusceptible (DS) and DR disease will be needed. Added to this, a massive scale-up in diagnosis and treatment of MDR and XDR TB was needed to prevent DR strains from becoming the dominant form of TB, something the government has actively begun. The WHO says that transmission in household and community settings is 'especially troubling' when it comes to children younger than 5 years of age, because they are highly susceptible to progression of active disease.

\section{Besides lives lost, cost alone must boost efforts}

Management of MDR TB is more complex, costly and time-consuming and less effective than is management of DS TB. One local study, also from Dheda's team, analysing the comparative 2011 US dollar costs of diagnosis and treatment of DS TB, MDR TB and XDR TB (based on and assuming adherence to the national SA TB guidelines and using published clinical outcome data), found that the per patient cost of XDR TB was $\$ 26392$, four times greater than MDR TB (\$6 772) and 103 times greater than DS TB (\$257). Despite DR TB comprising only $2.2 \%$ of the case burden, it consumed $32 \%$ of the total estimated national TB budget of $\$ 218$ million. The study also found that a decentralised XDR TB treatment programme could potentially reduce costs by $\$ 6930$ per case $(26 \%)$ and reduce the total amount spent on DR TB by $7 \%{ }^{[1]}$

While we 'have it bad' (incidence-wise), we are not alone. Dheda said that the highest TB burdens (by TB-infected numbers) were in India and China. The WHO observed in one of its latest reports: 'Responding to drug-resistant tuberculosis is possibly one of the most profound challenges facing global health.'

\section{Chris Bateman}

chrisb@hmpg.co.za

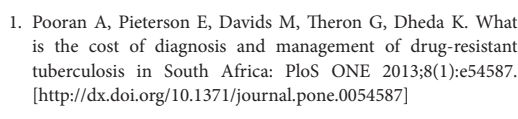

1. Pooran A, Pieterson E, Davids M, Theron G, Dheda K. What is the cost of diagnosis and management of drug-resistant tuberculosis in South Africa: PloS ONE 2013;8(1):e54587. [http://dx.doi.org/10.1371/journal.pone.0054587]

S Afr Med J 2014;104(8):528-529.

DOI:10.7196/SAMJ.8622 